\title{
Recurrent Hepatic Artery Thrombosis Following Living Donor Liver Transplant as Sequelae of SARS-CoV-2 Infection-a Case Report
}

\author{
Anupam Raj $^{1} \cdot$ Vijay Shankar $^{1}$ (1) Saurabh Singhal ${ }^{2} \cdot$ Neerav Goyal $^{2} \cdot$ Venuthurimilli Arunkumar $^{2}$. \\ Hitendra Kumar Garg ${ }^{3} \cdot$ Atish Pal $^{1}$
}

Accepted: 3 October 2021 / Published online: 8 October 2021

(c) The Author(s), under exclusive licence to Springer Nature Switzerland AG 2021

\begin{abstract}
As the second wave of COVID-19 disease is gripping the globe, liver transplant centers are increasingly receiving patients recovered from SARS-CoV-2 infection in recent few weeks. Unexpected complications in these patients are increasingly being recognized. We performed liver transplantation on a 51-year-old gentleman with decompensated liver disease 23 days after recovering from a mild SARS-CoV-2 infection. Surprisingly, despite massive blood loss and a prolonged anhepatic phase, his thromboelastographic (TEG) parameters persistently revealed hypercoagulability. After a brief uneventful early post-operative period, he developed hepatic arterial thrombosis on the 14th post-operative day, and again after 4 days, both of which required surgical intervention. Following discharge, the artery was thrombosed again which was only picked up when he developed a cholangiolar abscess, leading to graft loss necessitating re-transplantation. There is a lot of evidence suggesting that patients with SARS-CoV-2 infection tend to be hypercoagulable. We believe that this hypercoagulability might have played a significant role in the development of hepatic arterial thrombosis and eventual graft loss in this patient. This highlights the importance of revisiting anticoagulation protocols in liver transplant recipients recovered from COVID-19 and base them on TEG rather than routine parameters such as INR and APTT, which are routinely deranged in such patients.
\end{abstract}

Keywords Liver transplantation $\cdot$ SARS-CoV-2 $\cdot$ COVID-19 $\cdot$ Hypercoagulability $\cdot$ Arterial thrombosis

$\begin{array}{ll}\text { Abbreviations } & \\ \text { APSF } & \text { Anesthesia Patient Safety Foundation } \\ \text { aPTT } & \text { Activated partial thromboplastin time } \\ \text { ASA } & \text { American Society of Anesthesiologists } \\ \text { CLD } & \text { Chronic liver disease } \\ \text { CT } & \text { Computed tomography } \\ \text { HAT } & \text { Hepatic artery thrombosis }\end{array}$

Dr. Anupam Raj, Dr Vijay Shankar and Dr. Saurabh Singhal have equal contribution

This article is part of the Topical Collection on Covid-19

Vijay Shankar

vijaysnair95@gmail.com

1 Department of Anaesthesiology and Critical Care, Indraprastha Apollo Hospital, Sarita Vihar, New Delhi, India 110076

2 Liver Transplant and Hepato-Pancreatobiliary Surgery (LTHPS) Unit, Indraprastha Apollo Hospital, New Delhi, India

3 Department of Transplant Hepatology, Indraprastha Apollo Hospital, New Delhi, India

\begin{tabular}{|c|c|}
\hline INR & International normalized ratio \\
\hline LDH & Lactate dehydrogenase \\
\hline LFT & Liver function test \\
\hline LT & Liver transplant \\
\hline RT PCR & $\begin{array}{l}\text { Reverse transcriptase polymerase chain } \\
\text { reaction }\end{array}$ \\
\hline SARS-CoV-2 & $\begin{array}{l}\text { Severe acute respiratory syndrome corona- } \\
\text { virus } 2\end{array}$ \\
\hline TEG & Thromboelastogram \\
\hline
\end{tabular}

\section{Introduction}

As the rapid emergence of the second wave of COVID-19 pandemic shows no signs of slowing down [1], transplant clinicians all over the world are facing the scenarios of more and more SARS-CoV-2 recovered patients presenting for solid organ transplantation [2]. COVID-19 is still not fully understood with new symptoms, organ system involvements, and new complications emerging each day. Liver transplant (LT) recipients are a special population with several factors deciding the graft related and the overall outcome. There 
is a certain degree of uncertainty regarding the safety of performing liver transplantation in a patient who has recovered from SARS-CoV-2 infection. Not just the theoretical possibility of a flare-up of infection with immunosuppression, a worry which has been proven wrong as per several reports, there are several other variables at play which may affect the new liver graft. Vascular complications as seen in other patients may affect the liver grafts as well. It has become apparent that COVID-19 is a multisystem disease [3]; the extend of involvement varies significantly from patient to patient. When faced with a patient recovered from SARS-CoV-2 infection presenting for LT, clinicians are faced with a host of dilemmas including the latency of the virus, extend of systemic involvement, optimal timing of surgery, and appropriate post-operative immunosuppression. In this report, we describe the case of a patient with decompensated ethanolic chronic liver disease who underwent a living related donor hepatectomy after recovering from SARS-CoV-2 virus. This manuscript adheres to the CARE guidelines as appropriate for a case report.

\section{Description of the Case}

A 51-year-old male patient was admitted under our care in November 2020 with a diagnosis of ethanol-related chronic liver disease (CLD), decompensated with ascites and encephalopathy, and was evaluated for living related LT. He did not have any coagulation disorder on routine preoperative workup. A routine COVID-19 RT PCR sent as part of his evaluation came positive. Symptoms were mild in the form of fever and occasional cough. He was quarantined and was started on a 5-day course of azithromycin in addition to the supportive management, following which his symptoms subsided. A repeat COVID-19 RT PCR after 14 days turned out to be negative. After weighing the potential unknown risks following a recent SARS-CoV-2 infection and the deleterious effects of further decompensation of the liver disease, it was decided to postpone his transplantation for a period of 2 weeks.

Once he was readmitted for transplant, all the investigations were repeated which included the routine set of pretransplant tests as well as interleukin-6, lactate dehydrogenase (LDH), and ferritin. The preoperative investigations have been summarized in Table 1. A high-resolution computed tomography (CT) of chest was done to rule out any sequalae of COVID-19 pneumonia, which revealed small patchy ground glass opacities in the right lower lobe with no interstitial thickening, a CT score of $8 / 25$. The patient and his family members were thoroughly explained regarding the unknown risks of SARS-CoV-2 and the possible impact on graft-related outcome. After obtaining adequate consent from the patient as well as required clearances from a multidisciplinary clinical team, it was decided to go ahead with transplantation procedure 23 days after recovering from COVID-19 disease.

A conduction of anesthesia was done as per our standardized institutional protocol. The dissection phase was
Table 1 Blood investigations at the time of COVID-19 and before each transplant

\begin{tabular}{llllll}
\hline Investigations & $\begin{array}{l}\text { On admission } \\
\text { for COVID-19 }\end{array}$ & $\begin{array}{l}\text { 1 day before } \\
\text { first transplant }\end{array}$ & $\begin{array}{l}\text { 1 day before } \\
\text { retransplant }\end{array}$ & $\begin{array}{l}\text { 2 days after } \\
\text { retransplant }\end{array}$ & $\begin{array}{l}\text { 5th day after } \\
\text { retransplant }\end{array}$ \\
\hline 9th hemoglobin $(\mathrm{g} / \mathrm{dl})$ & 11.6 & 10.8 & 9.2 & 10.4 & 9.2 \\
WBC count $\left(/ \mathrm{mm}^{3}\right)$ & 5400 & 4300 & 6400 & 8200 & 11,000 \\
Platelet $\left(/ \mathrm{mm}^{3}\right)$ & 13,2000 & 150,000 & 18,0000 & 70,000 & 34,000 \\
INR & 1.9 & 2.1 & 1.9 & 2.2 & 2.8 \\
Urea $(\mathrm{mg} / \mathrm{dl})$ & 32 & 32 & 56 & 64 & 76 \\
Creatinine $(\mathrm{mg} / \mathrm{dl})$ & 0.8 & 1.0 & 0.9 & 0.8 & 0.9 \\
Total bilirubin $(\mathrm{mg} / \mathrm{dl})$ & 2.3 & 2.1 & 1.9 & 1.6 & 9.8 \\
AST $(\mathrm{U} / \mathrm{L})$ & 65 & 59 & 65 & 63 & 345 \\
ALT $(\mathrm{U} / \mathrm{L})$ & 62 & 35 & 43 & 45 & 324 \\
ALP $(\mathrm{U} / \mathrm{L})$ & 54 & 53 & 145 & 154 & 223 \\
Albumin $(\mathrm{g} / \mathrm{dl})$ & 3.5 & 3.2 & 3.2 & 2.9 & 2.6 \\
IL-6 $(\mathrm{pg} / \mathrm{ml})$ & 32 & 15 & & & \\
Ferritin $(\mathrm{ng} / \mathrm{ml})$ & 300 & 100 & 240 & & \\
D Dimer $(\mathrm{ng} / \mathrm{ml})$ & 450 & 220 & & & \\
Procalcitonin $(\mathrm{ng} / \mathrm{ml})$ & $<0.5$ & $<0.5$ & 0.6 & 9.8 & \\
Protein C $(\mathrm{IU} / \mathrm{dl})$ & & 80 & & & \\
Protein S $(\mu \mathrm{mg} / \mathrm{ml})$ & & 25 & & & \\
Antithrombin III $(\%)$ & & 95 & & &
\end{tabular}

$A L P$, alkaline phosphatase; $A L T$, alanine aminotransferase; $A S T$, aspartate aminotransferase; $I L-6$, interleukin-6; INR, international normalized ratio; $W B C$, white blood cells 
complicated by profuse surgical bleeding owing to dense adhesions. Packed red cells were transfused aiming at a hemoglobin level of $9 \mathrm{gm} / \mathrm{dl}$. The administration of platelets, plasma, and cryoprecipitate was based on the thromboelastogram (TEG) which was repeated 2-hourly. The intraoperative TEG readings are given in Table 2. Due to significant bleeding from dense perihepatic collaterals, recipient hepatectomy was done with porta-first approach, prolonging the anhepatic time. Despite significant bleeding and prolonged anhepatic time of $4 \mathrm{~h}$, it was noticed that the patient maintained a hypercoagulable TEG throughout the surgery, as evidenced by a coagulation index value of more than +3 . A total of 20 packed red cell units and 8 units of fresh frozen plasma were transfused intraoperatively. He received the modified right lobe graft (right lobe without middle hepatic vein), and segment 5 and segment 8 veins were reconstructed using a PTFE graft to fashion a neomiddle hepatic vein. Graft implantation was uneventful with standard hepatic vein and portal vein anastomoses. Graft had a single artery which was anastomosed with recipient's right hepatic artery (which originated from common hepatic artery) using interrupted $8-0$ prolene sutures. There was no tension, redundancy, or luminal discrepancy in arterial anastomosis. Recipient's left hepatic artery originated from left gastric artery (replaced left gastric artery-type 2 anatomy) and was ligated. The rest of the surgery was uneventful, and the patient was shifted to the post-transplant intensive care unit for further management.

Inotropic support was weaned off, and the patient was extubated on the 2nd post-operative day. Post-operative antibiotic therapy included cefoperazone-sulbactum, teicoplanin, metronidazole, and fluconazole. Low-dose immunosuppression was started on the evening of first post-operative day using tacrolimus and mycophenolate mofetil along with tapering doses of methylprednisolone which was later changed to oral prednisolone. Patient tolerated the incremental doses of immunosuppression well which was tailored according to the patients LFT's and tacrolimus trough levels. Patient did not receive heparin infusion during or in immediate post-operative period due to deranged prothrombin time-international normalized ratio (INR) and activated partial thromboplastin time (aPTT) ratio, both being more than 2, although all post-operative TEGs remained normal to hyper-coagulable.
Anticoagulation was started from the 3rd post-operative day in the form of low-molecular-weight heparin and was discontinued on the 10th post-operative day when aspirin $75 \mathrm{mg}$ once daily was initiated. Early post-operative course was satisfactory with near normalization of liver enzymes by the 7th day post transplantation. As per the protocol at our center, ultrasound doppler of liver graft was done twice daily for the first five post-operative days and then once daily from the sixth to tenth post-operative day; all doppler studies revealed normal inflows and outflows, homogenous graft, and no intra-hepatic biliary dilatation.

While patient's liver function tests showed recovery as expected, a repeat doppler done on the 14th day of transplantation revealed absent hepatic arterial flow. A CT angiography of the liver was done immediately which confirmed hepatic artery thrombosis (HAT). Digital subtraction angiography-guided thrombolysis/thrombectomy was attempted but was unsuccessful. The patient was taken for emergency re-exploration. Intraoperatively, the graft was homogenous with no signs of ischemia. Graft artery had no visible or palpable pulsations. The arterial anastomosis was disassembled. A fleshy thrombus was present in the recipient artery extending to the root of common hepatic artery. Thrombectomy of the right hepatic artery was done using a 2-Fr Fogarty catheter. Vascular patency was satisfactory after the procedure, and he was shifted to the intensive care unit and started on low-molecular-weight heparin for anticoagulation. Immediately, post exploration, his liver enzymes increased, which started coming down the subsequent days. Unfortunately, he again developed hepatic artery thrombosis on the 18th day of transplantation (4 days after re-exploration). He was taken up for a reexploration. Thrombectomy was done, and an autologous saphenous vein graft was used as conduit to anastomose the graft hepatic artery with left gastric artery. In view of the recurrent hepatic artery thrombosis, it was decided to initiate him on hyperbaric oxygen therapy. He received a total of 4 cycles of hyperbaric oxygen therapy which were uneventful. By the end of the 27th day post-transplant, he had near normalization of LFTs, normal inflows and outflows of the graft and hence he was discharged on the 30th post-operative day continuing low-molecular-weight heparin and with advices for a strict follow-up.
Table 2 Intraoperative TEG values

\begin{tabular}{lllllll}
\hline & $R(\min )$ & $K(\min )$ & $\alpha\left(^{\circ}\right)$ & MA $(\mathrm{mm})$ & LY 30 (\%) & CI \\
\hline Dissection phase & 3.9 & 2.1 & 72 & 76 & 1.2 & 6.32 \\
Anhepatic phase & 4.4 & 1.5 & 72 & 70 & 0.7 & 5.3 \\
Neo-hepatic phase & 3.6 & 1.2 & 80 & 73 & 1.1 & 5.5 \\
\hline
\end{tabular}

$\alpha$, alpha or the angle; $C I$, coagulation index; $K$, kinetic value; $L Y 30$, lysis after $30 \mathrm{~min} ; M A$, maximum amplitude; $R$, reaction time; $T E G$, thromboelastogram 
On the 18th day post discharge, he presented to our hospital with complaints of fever and chills. Abdominal imaging revealed the presence of a cholangiolar abscess (Fig. 1) with absent hepatic artery flow yet again. He was admitted and initiated on broad spectrum antibiotics. The blood investigations revealed leucocytosis and transaminitis (Table 1). Initially, he was managed with percutaneous drainage of the major abscesses. However, with persistent transaminitis and graft abscess, the patient and his family were counselled regarding the loss of graft and need for a retransplant after control of sepsis, to which he consented. Identifying and evaluating a new donor took us 2 weeks during which we were able to control his infection. He was taken up for retransplantation 70 days after the initial transplantation.

Surgery for re-transplantation was eventful, requiring massive blood transfusion and inotropic support. Once in the ICU, we encountered great difficulty in weaning him off inotropes due to persistent vasoplegia as evidenced by the cardiac output monitor and echocardiogram. His liver enzymes and bilirubin started increasing by the 3rd postoperative day following a brief period of 2 days where the enzymes seemed to touch baseline. As there were no signs of infection or any vascular and biliary complication, we decided to treat it as acute cellular rejection using steroid pulses and antithymocyte globulin after performing a liver biopsy.

His clinical condition failed to improve with further worsening of hemodynamics and liver function tests. His liver biopsy revealed centrilobular necrosis with cholestasis and no evidence of inflammatory infiltrate. We rationalized the drugs and performed 2 cycles of plasmapheresis keeping in mind the possibility of acute cellular rejection along with drug-induced liver injury. In spite of our best efforts, his clinical condition deteriorated with the development of multiorgan failure. He suffered an irreversible cardiac arrest 80 days after the initial surgery.

\section{Discussion}

COVID-19 recovered patients presenting for liver transplantation are going to be increasingly frequent in the coming years. There is limited evidence regarding the sequalae of SARS-CoV-2 and its impact on perioperative recovery [3]. The ASA-APSF joint statement on elective surgery and anesthesia for patients after COVID-19 infection suggested a waiting period of 4 weeks after recovery in case of asymptomatic or mildly symptomatic patients [4]. Other major surgical and anesthesia associations have also given similar protocol [5]. Even though, these guidelines were not available at the time of our case, we had ensured a complete clinical and radiological recovery along with normal
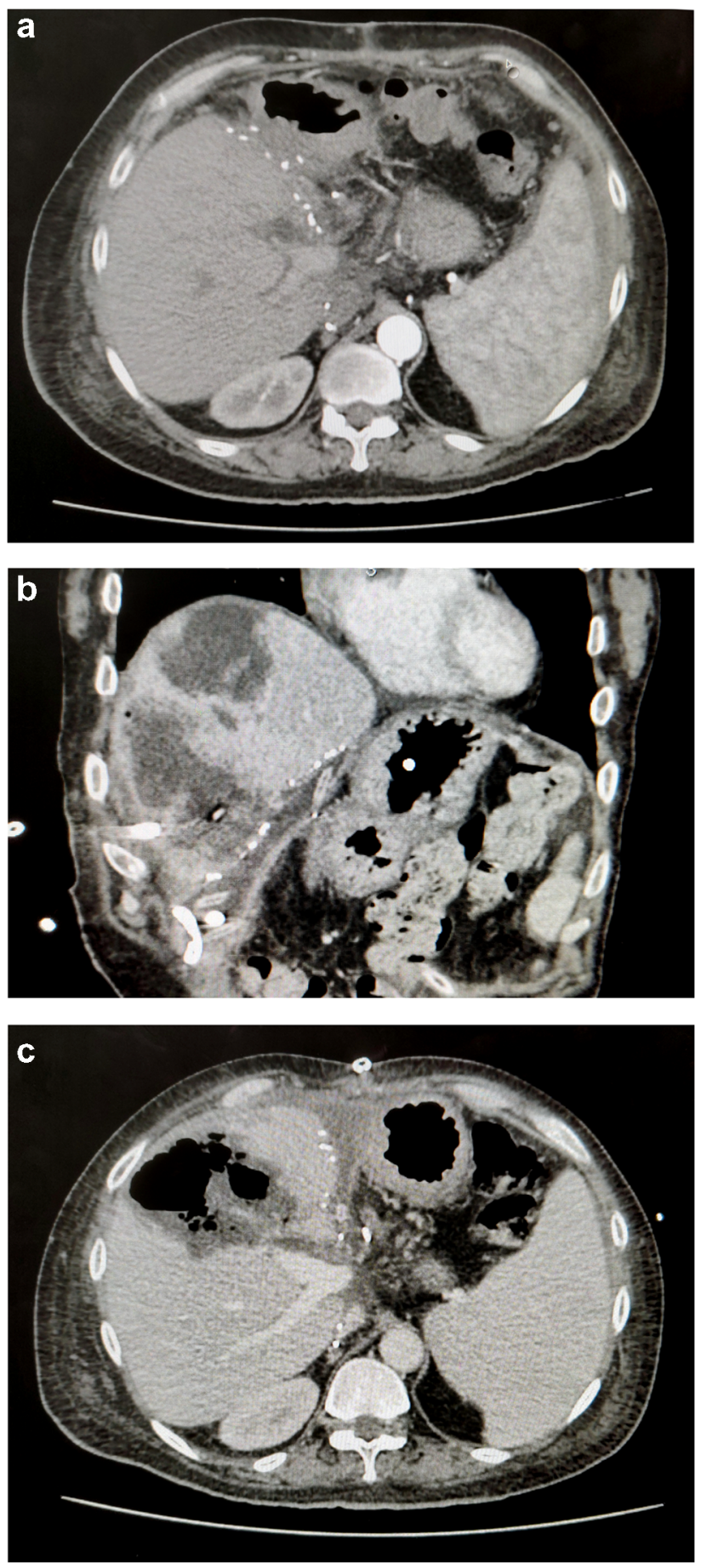

Fig. 1 a Arterial phase of CT angiography on the 14th post-operative day of first liver transplant showing no intrahepatic arterial filling. b Developing abscess at the time of readmission. These abscesses were drained through percutaneous pigtail drain placement. c Abscess cavity with graft loss (majorly anterior sector) before second liver transplant 


\begin{tabular}{|c|c|c|c|c|c|c|c|c|c|c|c|}
\hline Days & -37 & -23 & -2 & 0 & 2 & 14 & 18 & 30 & 48 & 70 & 80 \\
\hline $\begin{array}{l}\text { Clinical } \\
\text { Events }\end{array}$ & $\begin{array}{l}\text { Diagnosed } \\
\text { COVID-19+ }\end{array}$ & $\begin{array}{c}\text { First } \\
\text { RT PCR } \\
\text { negative }\end{array}$ & $\begin{array}{c}\text { Second } \\
\text { RT PCR } \\
\text { negative }\end{array}$ & First LDLT & Extubated & First HAT & $\begin{array}{l}\text { Second } \\
\text { HAT }\end{array}$ & Discharged & $\begin{array}{c}\text { Admitted } \\
\text { with HAT+ } \\
\text { Abscess }\end{array}$ & $\begin{array}{l}\text { Redo } \\
\text { LDLT }\end{array}$ & Death \\
\hline
\end{tabular}

Fig. 2 Graphical timeline of the critical events. HAT, hepatic artery thrombosis; LDLT, living donor liver transplant; RT PCR, reverse transcriptase polymerase chain reaction

inflammatory markers such as IL-6, D-Dimer, and ferritin at the time of transplant.

Hypercoagulability [6], reactivation of latent virus with immunosuppression, and occult multi-systemic sequalae of COVID-19 were our main concerns in this patient. Fortunately, in spite of intense immunosuppression, we did not find any clinical or molecular evidence of SARS-CoV-2 in the post-operative period. However, the first liver transplantation in this patient was complicated due to the repeated occurrence of hepatic arterial thrombosis during the postoperative period, which ultimately leads to a loss of the transplanted graft. Even though technical aspects might have contributed to the occurrence of HAT, we do believe that hypercoagulability did play a significant role (Fig. 2).

There is a growing pool of data which suggests patients infected by SARS-CoV-2 virus tend to be hypercoagulable [7, 8]. Many reports have come with evidence of acute arterial thrombosis in patients infected with SARS-CoV-2. Thromboelastogram is a better predictor of coagulation parameters than the conventional parameters, such as INR and aPTT [9]. Thromboelastographic studies have demonstrated significant hypercoagulopathy in a majority of these patients [10]. This was along the lines of our experience in this case where the patient demonstrated significant hyper coagulopathy in spite the presence of clinically significant bleeding. It is interesting to note that duration of hypercoagulability post COVID-19 infection is not well known, and based on our experience, it may well persist to more than 4 weeks post infection.

We now believe that the patient should have been started on novel anticoagulants after discontinuing heparin on the 10th post-operative day. Novel anticoagulants are well tolerated orally and have rapid onset and offset of action along with fewer drug interactions and predictable pharmacokinetics [11]. Probably, demonstration of hypercoagulability by a thromboelastogram preoperatively would have helped in better management of anticoagulation in this case. Starting unfractionated heparin intraoperatively might be a good idea in such cases but was not done in our case as the patient had clinically significant bleeding.

Our patient developed acute cellular rejection post operatively after the re transplantation. Dhand et al. reported a similar experience with a COVID-19 recovered patient presenting for liver transplantation which was successfully managed by high-dose steroids and $\mathrm{T}$ cell depletion [2]. In our case, we were unable to successfully manage the graft dysfunction in spite of early initiation of intense immunosuppression by means of steroid pulses, anti-thymocyte globulin, and plasmapheresis. The presence of cholestasis in the liver biopsy indicates the presence of DILI in addition to acute cellular rejection. This was probably the result of a prolonged course of antibiotics following the development of cholangiolar abscess which continued well into the second post-operative course as well.

We believe that our experience will throw some valuable insights about managing liver transplant in a patient recovered from SARS-CoV-2 infection. This was the first case of graft loss after nearly 400 cases in our institute in the last 4 years. Significant hypercoagulability probably had a significant impact in the development of hepatic arterial thrombosis in this patient and subsequent loss of graft. Keeping in mind the pandemic proportions and the ambiguity regarding the effects of SARS-CoV-2, thorough multi-systemic evaluation should be performed even for asymptomatic COVID-19 recovered patients presenting for solid organ transplantation.

\section{Conclusion}

It's well known that patient tend to be hypercoagulable during COVID infection. However, it is unclear how long the hypercoagulability will persist following recovery from COVID infection. We believe that this hypercoagulability might have played a significant role in the adverse outcomes in this case. In the light of our above experience, it may be prudent to base anticoagulation parameters on global visco elastic tests like TEG rather than PT INR and APTT.

Author Contribution Each individual listed as an author on this manuscript contributed substantially and in accordance with the guidelines of the International Committee of Medical Journal Editors. Individual contributions are listed as under the following:

Anupam Raj: Management of the case, drafting, and editing the article

Vijay Shankar: Assistance in managing the case, drafting the article 
Saurabh Singhal: Assistance in managing the case, editing the article

Neerav Goyal: Assistance in managing the case, editing the article Arun Venuthurimilli: Editing and drafting the article

Hitendra Garg: Editing and drafting the article

Atish Pal: Assistance in managing the case

Data Availability Clinical data of the patient will be available if requested.

\section{Declarations}

Ethics Approval Approval from the institutional ethical committee has been obtained prior to drafting of the manuscript.

Consent to Participate Not applicable

Consent for Publication Has been obtained

Conflict of Interest The authors declare no competing interests.

\section{References}

1. WHO Coronavirus (COVID-19) Dashboard [Internet]. [cited 2021 Apr 25]. Available from: https://covid19.who.int

2. Dhand A, Bodin R, Wolf DC, Schluger A, Nabors C, Nog R, et al. Successful liver transplantation in a patient recovered from COVID-19. Transpl Infect Dis. 2021;23(2):e13492.

3. Bui N, Coetzer M, Schenning KJ, O'Glasser AY. Preparing previously COVID-19-positive patients for elective surgery: a framework for preoperative evaluation. Perioper Med (Lond). 2021;10(1):1.
4. ASA and APSF joint statement on elective surgery and anesthesia for patients after COVID-19 infection [Internet]. [cited $2021 \mathrm{Apr}$ 25]. Available from: https://www.asahq.org/about-asa/newsroom/ news-releases/2020/12/asa-and-apsf-joint-statement-on-electivesurgery-and-anesthesia-for-patients-after-covid-19-infection

5. Olson MT, Triantafyllou T, Singhal S. Resumption of elective surgery during the COVID-19 pandemic: what lessons can we apply? Eur Surg. 2020;5:1-3.

6. Abou-Ismail MY, Diamond A, Kapoor S, Arafah Y, Nayak L. The hypercoagulable state in COVID-19: incidence, pathophysiology, and management. Thromb Res. 2020;194:101-15.

7. Osilli D, Pavlovica J, Mane R, Ibrahim M, Bouhelal A, Jacob S. Case reports: mild COVID-19 infection and acute arterial thrombosis. J Surg Case Rep. 2020;2020(9):rjaa343.

8. Al Mahruqi G, Stephen E, Abdelhedy I, Al Wahaibi K. Our early experience with mesenteric ischemia in COVID-19 positive patients. Ann Vasc Surg. 2021;73:129-32.

9. Raj A, Shankar V, Singhal S, Dhar P, Krishnan L, Goyal N, et al. Thromboelastogram demonstrates significant hypercoagulability in patients undergoing right donor hepatectomy-a prospective single-center analysis. Int J Transplant Res Med. 2020;15:6.

10. Bocci MG, Maviglia R, Consalvo LM, Grieco DL, Montini L, Mercurio G, et al. Thromboelastography clot strength profiles and effect of systemic anticoagulation in COVID-19 acute respiratory distress syndrome: a prospective, observational study. Eur Rev Med Pharmacol Sci. 2020;24(23):12466-79.

11. Tang N, Bai H, Chen X, Gong J, Li D, Sun Z. Anticoagulant treatment is associated with decreased mortality in severe coronavirus disease 2019 patients with coagulopathy. J Thromb Haemost. 2020;18(5):1094-9.

Publisher's Note Springer Nature remains neutral with regard to jurisdictional claims in published maps and institutional affiliations. 